ejection fraction (EF) increased. In patients, LVEDV and LVESV decreased on exercise, with an increase in LVSV and LVEF. RVEDV increased in size with a trend for ESV to be higher than at rest. RVSV and RVEF remained unchanged. Between groups, at rest, LVESV and LVSV were lower in patients with no significant differences in RV function. However, on exercise, patients had a significantly higher RVESV and lower RVSV and RVEF, compared to HCs. Conclusion: Exercise CMR is a sensitive, accurate test which can determine physiological changes in RV and LV function. We have shown that on exercise, when compared to $\mathrm{HC}$, the $\mathrm{LV}$ in patients with iPAH decreases in size, the RV increases in size and RV dysfunction becomes apparent which was otherwise not present at rest. This could potentially be valuable in the assessment of response to treatment and prognosis in patients with iPAH.

\section{THE ROLE OF CARDIAC MAGNETIC RESONANCE IMAGING IN PATIENTS WITH CARCINOID HEART DISEASE}

Boyang Liu*, Manvir Hayer, Shanat Baig, Tahir Shah, Stephen Rooney, Nicola Edwards, Richard Steeds. Queen Elizabeth Hospital Birmingham; *Presenting Author

\subsection{6/heartjnl-2016-309890.126}

Introduction Carcinoid heart disease (NET-CHD) is a frequent and adverse complication of carcinoid syndrome due to right ventricular (RV) failure. Medical therapy alone has a 2-year survival of approximately $20 \%$ and while surgical valve replacement is effective in improving symptoms and may increase survival, peri-operative risk remains approximately 15-20\%. Transthoracic echocardiography (TTE) is considered the gold standard for assessment of NET-CHD and data on the role of cardiac magnetic resonance imaging (CMR) are limited despite recognised advantages in assessment of the right heart. The aim of this study was to assess the role of CMR in assessment of NET-CHD.

Methods This is a retrospective cohort study of 50 consecutive patients with proven NET referred with elevated NT pro-BNP to the European Centre of Excellence for Neuroendocrine Tumours in Birmingham between 2005-2015. At referral, all subjects underwent comprehensive left ventricular (LV) and RV assessment with CMR (1.5T Siemens Avanto), including deformation (Tissue Tracking, cvi42 ${ }^{\circledR}$ Circle Cardiovascular Imaging), and late gadolinium enhancement (LGE).

Results In total, 36 patients were diagnosed with NET-CHD and 14 without (CHD-neg). Right sided valve disease was universal in NET-CHD: severe tricupid regurgitation (97\%), severe pulmonary regurgitation (86\%). On CMR, RV end-diastolic volume (EDV) and end-systolic volume (ESV) were increased $\left(120 \pm 30 \mathrm{ml} / \mathrm{m}^{2}\right.$ vs. $67 \pm 14 \mathrm{ml} / \mathrm{m}^{2}, \mathrm{p}<0.01 ; 49$ $\pm 20 \mathrm{ml} / \mathrm{m}^{2}$ vs. $\left.11 \pm 3 \mathrm{ml} / \mathrm{m}^{2}, \mathrm{p}<0.01\right)$ but with no difference in RVEF $(60 \pm 14 \%$ vs. $60 \pm 9 \% p=0.92)$. There was early evidence of ventricular-ventricular interaction, with reduction in both $\operatorname{LVEDV}\left(53 \pm 16 \mathrm{ml} / \mathrm{m}^{2}\right.$ vs. $72 \pm 16 \mathrm{ml} /$ $\left.\mathrm{m}^{2}, \mathrm{p}<0.01\right)$ and $\operatorname{LVESV}\left(19 \pm 10 \mathrm{ml} / \mathrm{m}^{2}\right.$ vs. $28 \pm 16 \mathrm{ml} /$ $\left.\mathrm{m}^{2}, \mathrm{p}<0.05\right)$ in NET-CHD, but no difference in LV ejection fraction $(67 \pm 8 \%$ vs. $63 \pm 14 \%, p=0.3)$. There was no difference in LV global longitudinal strain (GLS) or circumferential strain (GCS) between groups. RV LGE indicative of endocardial plaques was present in 6/36 (17\%) but not observed in CHD-neg. Diffuse LV LGE was present 5 NET-
CHD patients. Over follow up (median 1.3 years [0.6-3.1]), 20 patients with NET-CHD died. These patients had a lower GCS $(14.8 \pm 4.6 \%$ vs. $18.2 \pm 4.4 \%, \mathrm{p}<0.05)$ and lower GLS $(14.7 \pm 4.7 \%$ vs. $18.3 \pm 4.4 \%, \mathrm{p}<0.05)$ on CMR but no difference in LVEF, RVEF, LV volumes, RV volumes or NT-proBNP. In a logistic regression model, LV GLS remained an independent predictor of death.

Conclusion Significant increase in RV size and the presence of RV plaques are measurable on CMR early following referral with NET-CHD. This is sufficient to adversely affect LV filling and global deformation, which may contribute to effort intolerance and adverse cardiovascular outcomes.

\section{SERIAL MONITORING OF LEFT VENTRICULAR EJECTION FRACTION IN PATIENTS WITH HAEMATOLOGICAL MALIGNANCIES RECEIVING ANTHRACYCLINE-BASED CHEMOTHERAPY}

Polyvios Demetriades*, Emily Ho. Sandwell and West Birmingham NHS Hospitals Trust; *Presenting Author

\subsection{6/heartjnl-2016-309890.127}

Introduction Anthracyclines are one of the most widely used chemotherapeutic agents. However, their use has been limited due to significant risk of cardiotoxicity. This has been reported in $6.2 \%$ of adult patients with haeamatological malignancies. Early-onset cardiotoxicity is by far the commonest, occurs within the first year following treatment and manifests as a form of dilated cardiomyopathy. Early detection with imaging, even in asymptomatic patients, and appropriate treatment with anti-failure medication leads to recovery of left ventricular function (LVEF) in up to $82 \%$ of patients. This highlights the need for a consensus strategy to guide monitoring frequency and duration in the long-term management of these patients. We conducted a quality improvement project to review the current practice in the Haematology department of our Trust.

Methods We identified patients with haematological malignancies that were commenced on anthracycline-based chemotherapy regimens between March 2014 and August 2015.

We collected data regarding pre and post-chemotherapy imaging with echocardiograms and compared our findings against the recommendations of the "ESMO Clinical Practice Guidelines". These suggest that all patients should have serial monitoring of their LVEF at baseline and at 3-monthly intervals for the first year post-treatment.

In addition, we performed a qualitative analysis to review the management of the patients with abnormal echocardiograms both pre and post-chemotherapy.

Results We identified a total of 83 patients, of which $74.7 \%$ were treated with doxorubicin-based chemotherapy.

The majority of patients $(96.4 \%)$ had a baseline echocardiogram to assess LVEF prior to chemotherapy. However, only 29 patients $(34.9 \%)$ had imaging post-chemotherapy. Unfortunately, in the majority $(\mathrm{n}=18)$ this was requested due to symptoms rather than as part of serial monitoring.

Of our cohort, six patients had a degree of left ventricular systolic dysfunction (mild to moderate) prior to chemotherapy but proceeded without any cardiology input. Of these, only $50 \%$ had an echocardiogram post-chemotherapy. 


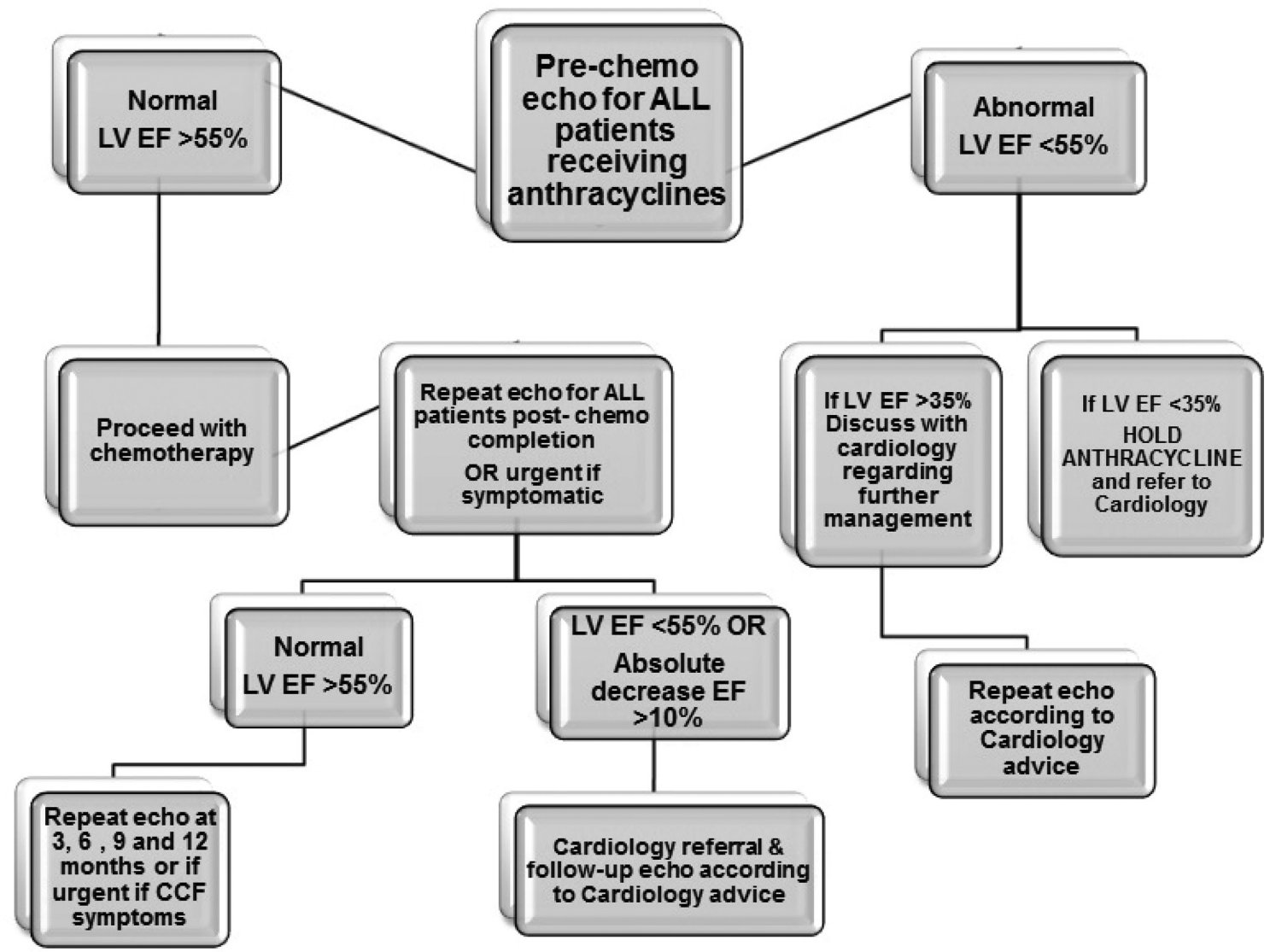

Abstract 127 Figure 1 Algorithm for the serial monitoring of LVEF in tpatients receiving anthracycline-based chemotherapies

In total, three patients developed impairment of left ventricular function post-chemotherapy due to anthracycline cardiotoxicity.

Discussion and conclusion We identified a significant lack of awareness on the monitoring strategies of patients receiving anthracycline-based chemotherapy possibly reflecting the lack of local guidelines. As a result, we introduced an algorithm that will guide the management of these patients (Fig 1).

In conclusion, our project has highlighted an area of clinical practice that requires effective communication and collaboration between different specialties. We strongly encourage clinicians to review their local practices and implement strategies to allow early identification and management of anthracycline-induced cardiotoxicity to prevent further morbidity and mortality.

\section{ABNORMAL GLOBAL LONGITUDINAL STRAIN IS ASSOCIATED WITH ALL-CAUSE MORTALITY IN HAEMODIALYSIS PATIENTS}

${ }^{1}$ Diana Chiu, ${ }^{2}$ Darren Green, ${ }^{2}$ Philip Kalra, ${ }^{2}$ Nik Abidin*. ${ }^{1} \mathrm{NHS}$; ${ }^{2}$ Salford Royal Hospital; *Presenting Author

\subsection{6/heartjnl-2016-309890.128}

Introduction Cardiovascular mortality is high in end-stage renal disease patients undergoing haemodialysis (HD). Early detection of cardiac dysfunction is important. Left ventricular global longitudinal strain (GLS) measures the maximal shortening of myocardial longitudinal length during systole compared to the resting length in diastole. Reduced GLS may reflect abnormal systolic function before loss of ejection fraction (EF) becomes apparent. We aimed to determine the prevalence, clinical correlates and prognostic value of abnormal GLS in stable HD patients.

Methods Clinical and echocardiographic data were obtained in a prospective study of HD patients at one centre. Survival analysis for GLS was performed using Cox regression adjusted for age, co-morbidities, dialysis chronicity, laboratory data, left ventricular mass index adjusted for height $\left(\mathrm{LVMIHt}^{2.7}\right)$ and Teicholz EF.

Results 199 patients had adequate speckle tracking images; the mean age was $62 \pm 14$ years, $69 \%$ were male, $39 \%$ had diabetes, $29 \%$ heart failure, $17 \%$ coronary artery disease. The mean GLS was $-13.4 \pm 3.5 \%$, LV ejection fraction (LVEF) $63.8 \pm 12.9 \%$ and LVMIHt $^{2.7} 53.6 \pm 17.2 \mathrm{~g} / \mathrm{m}^{2.7} .98 \%$ of patients had abnormal GLS (>-20\%), compared with $14 \%$ with reduced $\operatorname{LVEF}(<50 \%)$ and $55 \%$ with $\mathrm{LV}$ hypertrophy. Factors associated with an abnormal GLS included $\mathrm{LVMIHt}^{2.7}$ (OR 1.06, 95\% CI 1.04-1.09, P < 0.01), LVEF (OR 0.96, 95\% CI $0.94-0.99, \mathrm{P}<0.01$ ) and diabetes (OR 2.04, 95\% CI $1.08-3.9, p=0.03)$. Median follow-up was 24 (17-30) months, during which there were 41 deaths (21\%). After adjustment for age, diabetes, coronary artery disease, LVEF, LVMIHt ${ }^{2.7}$, 3 month-averaged serum potassium and albumin, a less negative GLS remained an independent predictor of allcause mortality (HR 1.18 for each 1\% worsening change in GLS, 95\% CI 1.03-1.35, P = 0.02). Figure 1.

Conclusions Abnormal GLS is highly prevalent amongst HD patients, and appears to be a better marker of all-cause 